\title{
A multipurpose voltage controlled oscillator
}

\section{A. V. PAWLOWSKI and D. R. LAWS \\ Atascadero State Hospital, Atascadero, California 93422}

This report describes a multipurpose voltage-controlled oscillator that is inexpensive, flexible, and adaptable to a variety of experimental purposes. Design features include the capability to (1) select a wide range of frequencies for experimental use, and (2) use the output to drive other digital circuits.

One of the more useful pieces of instrumentation in biofeedback experiments is the voltage-controlled oscillator, an instrument capable of providing continuous auditory feedback. The device described here is an inexpensive solid state oscillator that provides reliable and flexible operation.

\section{CIRCUIT DESCRIPTION}

The input stage (see. Fig. 1) is an integrated circuit operational amplifier used as a unity gain voltage follower. It provides high input impedance and, by adjusting $\mathrm{R} 4$, it can compensate for an input offset of $\pm 12 \mathrm{~V}$.

Q1 draws its base current from ICl through Limiting Resistor R3. The base current of Q1 determines the resistance between the collector and emitter of Q1. This, in turn, controls the charging rate of $\mathrm{Cl}$ in parallel with the external capacitor and, thus, the frequency of the unijunction transistor.

The signal from the emitter of the unijunction is amplified by Q3 and Q4 to prevent overloading of the unijunction. The output of Q4 is a ramp voltage, and it is used to drive Schmitt trigger Q5 and Q6. R12

*The opinions or conclusions stated in this paper are those of the authors and are not to be construed as official or as necessarily reflecting the policy of the Department of Health of the State of California. determines the point on the ramp at which the Schmitt trigger fires. Q7 and Q8 amplify the output of the Schmitt trigger, providing a final pulse output that has a $-12 \cdot \mathrm{V}$ "off" state and a $0-\mathrm{V}$ "on" state, the same frequency as that of the unijunction, and a pulse width determined by R12. The rise time is less than $1 \mu \mathrm{sec}$, making the output suitable for driving other digital equipment.

\section{OPERATION}

In operation, an external capacitor ( $\mathrm{C}$ ext) is chosen to provide a suitable range of frequencies (see Fig. 2) for the particular job at hand. The base line or reference voltage is then applied to the input and R4 is adjusted for any convenient reference frequency at the output. Any increase in voltage will then cause a corresponding increase in the output frequency. Inspection of Fig. 2 shows that the output frequency is a fairly linear function of input voltage. Using R12, the pulse width can be set as desired.

The output can be used to drive headphones, other digital equipment, or amplified to drive a speaker.

\section{APPLICATION}

This oscillator has been used in penile plethysmography (Laws \& Pawlowski, 1973a,b). Ss were provided with an audio signal that varied in proportion to the amount of penile engorgement. The purpose was to treat certain sexual disorders, for example, male patients confined for sexual offenses such as rape, child molestation, or exhibitionism. The Ss, with the assistance of biofeedback, were differentially trained to respond sexually in the presence of appropriate stimuli (e.g., adults) and to suppress the penile response in the presence of inappropriate ones (e.g., children). Of
Fig. 1. Circuit diagram of multipurpose voltage-controlled oscillator. Parts List: ICI = Sylvania ECG 941, National LM 741CH, or Motorola MC 1741CG (NOTE: pin numbers of $\mathrm{ICl}$ refer to the case style of the indicated components); Q1, Q5, Q6, Q8 = ECG 100; $Q 7=$ ECG 101; Q3, Q4 = ECG 102; Q2 = Sylvania ECG 6401 or Motorola HEP 310; $\mathrm{C} 1=.001$ microF; $\mathrm{C} 2=$ $220 \mathrm{pF} ; \mathrm{R} 1, \mathbf{R} 2=1$ megohm; $\mathrm{R} 3=$ $470 \mathrm{~K}$ ohms; $\mathrm{R} 4=10 \mathrm{~K}$ ohms; $\mathrm{R5}=$ 270 ohms; R6, R14 = 470 ohms; R7, R9, $R 15=620$ ohms; $R 8=2 \mathrm{~K}$ ohms; R10, R11 $=3.9 \mathrm{~K}$ ohms; R12 = Allen Bradiey Type RP102M, Spectrol Type 949-2-1, or Burns Type 275-1-102, all $1 \mathrm{~K}$ ohm, 20 turn; R13 $=150 \mathrm{ohms} ; \mathrm{R} 16=1 \mathrm{~K}$ ohm.

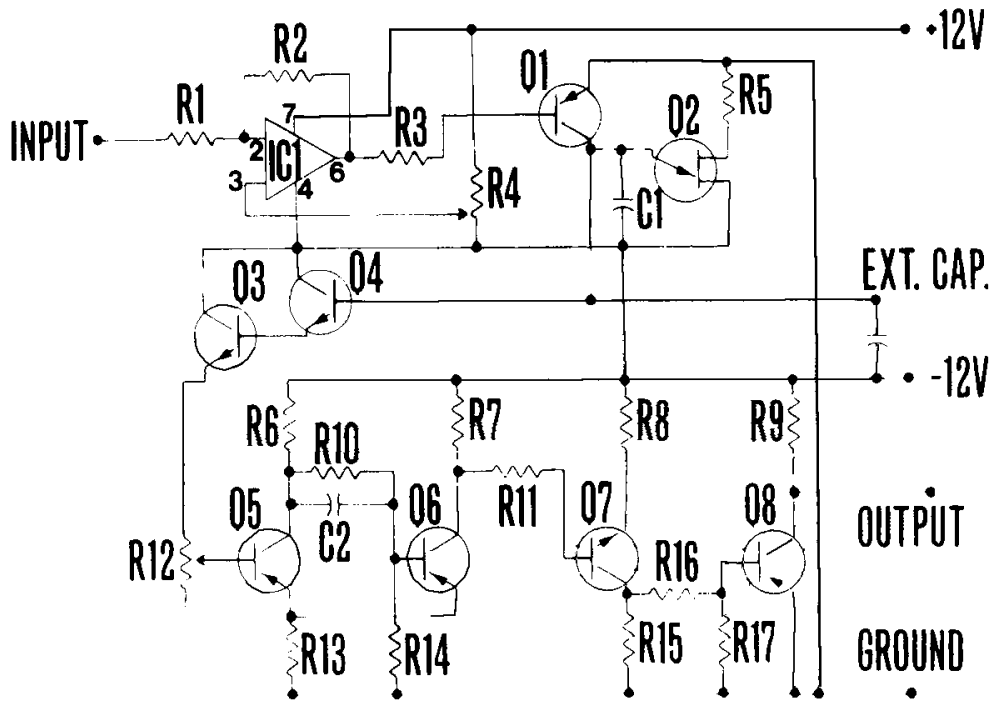



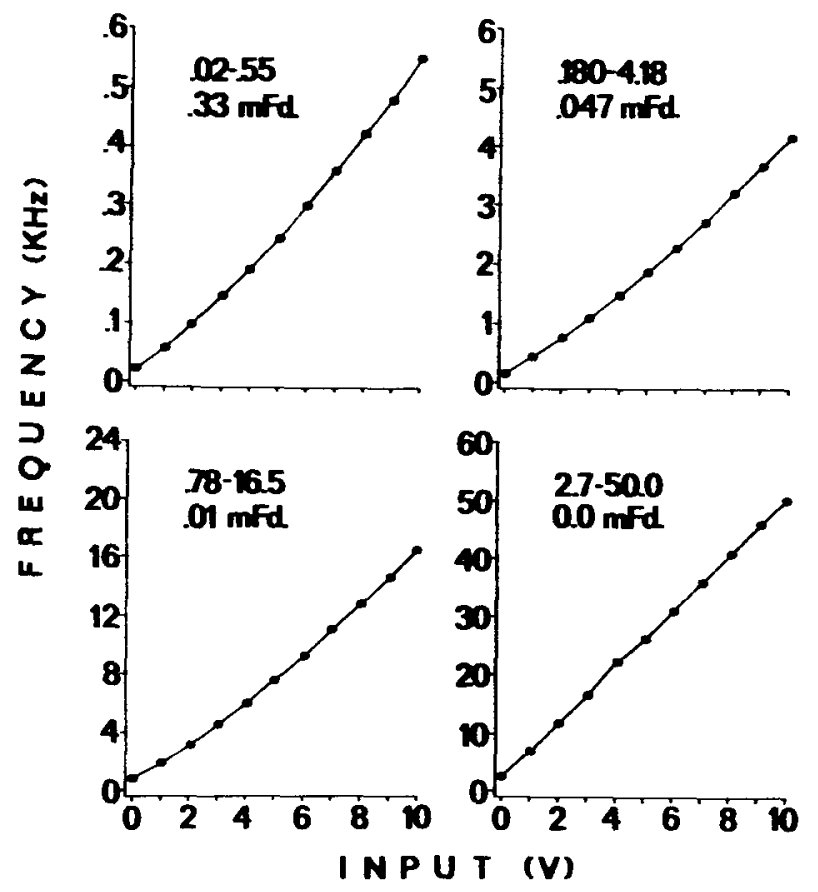

Fig. 2. Output frequency as a function of input voltage for four values of external capacitance.

course, the device would also be useful in biofeedback of other physiological systems.

\section{CONCLUSION}

The multipurpose voltage-controlled oscillator described here permits selection of a wide range of frequencies for experimental use and provides an output suitable for driving other digital circuits. This allows the investigator to tailor a frequency range that produces the best results in a particular $S$ or that best suits his experimental requirements.

\section{REFERENCES}

Laws, D. R. \& Pawlowski, A. V. The application of a multi-purpose biofeedback device to penile plethysmography. Journal of Behavior Therapy \& Experimental Psychiatry, $1973 \mathrm{a}$, in press.

Laws, D. R. \& Pawlowski, A. V. Unpublished data, Atascadero State Hospital, Atascadero, California, $1973 \mathrm{~b}$
(Received for publication November 15, 1973: revision received November 27, 1973.) 